\title{
Interaction Design in Digital Libraries
}

\author{
Organiser: Dr. Constantine Stephanidis \\ Institute of Computer Science (ICS) \\ Foundation for Research and Technology - Hellas (FORTH) \\ Science and Technology Park of Crete \\ GR - 71110 Heraklion, Crete, Greece \\ Tel: +30 - 81 - 391741 \\ Fax: $+30-81-391740$ \\ email: cs@ics.forth.gr
}

\begin{abstract}
In recent years, the field of Human-Computer Interaction (HCI) has made significant advances, penetrating an increasing number and range of computer-mediated human activities. In this context, interaction design has become a critical component of advanced interactive applications and telematic services as well as an increasingly complex challenge to meet.
\end{abstract}

This panel is concerned with interaction design in the domain of Digital Libraries (DL) and aims to bring together individuals interested in the interrelationships between $\mathrm{HCI}$ and $\mathrm{DL}$, to exchange experience, to advance an understanding and to promote common ground regarding the design, development and deployment of interactive DL services that meet the diverse abilities, skills, requirements, and preferences of individual users.

In particular, the panel seeks to broadly address and discuss a number of related themes, including:

- how interaction with DL services can be designed so as to meet the desirable levels of quality in use;

- alternative approaches to the development of user interfaces to DLs that exhibit task aware, Co-operative and intelligent behaviour;

- development of an understanding of how user interface software technologies (e.g. user modelling components, knowledge based user interface development toolkits, etc) can be integrated into architectural models for DLs.

Panellists

- Dr. Constantine Stephanidis, FORTH-ICS, GREECE (chair)

- Prof. David Benyon, Napier University, UK

- Dr. Mark Maybury, MITRE Corporation, USA

- Dr. Daniel Dardailler, W3C/WAI, FRANCE

C. Nikolaou, C. Stephanidis (Eds.): ECDL'98, LNCS 1513, p. 703, 1998.

(C) Springer-Verlag Berlin Heidelberg 1998 